\title{
High fluoride and low calcium levels in drinking water is associated with low bone mass, reduced bone quality and fragility fractures in sheep
}

\author{
M. J. K. Simon • F. T. Beil • W. Rüther • B. Busse • \\ T. Koehne $\cdot$ M. Steiner $\cdot$ P. Pogoda $\cdot$ A. Ignatius • \\ M. Amling $\cdot$ R. Oheim
}

Received: 28 May 2013 / Accepted: 28 February 2014 / Published online: 29 April 2014

(C) The Author(s) 2014. This article is published with open access at Springerlink.com

\begin{abstract}
Summary Chronic environmental fluoride exposure under calcium stress causes fragility fractures due to osteoporosis and bone quality deterioration, at least in sheep. Proof of skeletal fluorosis, presenting without increased bone density, calls for a review of fracture incidence in areas with fluoridated groundwater, including an analysis of patients with low bone mass.

Introduction Understanding the skeletal effects of environmental fluoride exposure especially under calcium stress remains an unmet need of critical importance. Therefore, we studied the skeletal phenotype of sheep chronically exposed to highly fluoridated water in the Kalahari Desert, where livestock is known to present with fragility fractures.

Methods Dorper ewes from two flocks in Namibia were studied. Chemical analyses of water, blood and urine were executed for both cohorts. Skeletal phenotyping comprised micro-computer tomography ( $\mu \mathrm{CT})$, histological, histomorphometric, biomechanical, quantitative backscattered electron imaging (qBEI) and energy-dispersive X-ray (EDX) analysis. Analysis was performed in direct comparison with undecalcified human iliac crest bone biopsies of patients with fluoride-induced osteopathy.
\end{abstract}

M. J. K. Simon • F. T. Beil • B. Busse • T. Koehne • P. Pogoda •

M. Amling $(\bowtie) \cdot R$. Oheim

Department of Osteology and Biomechanics, University Medical

Center Hamburg-Eppendorf, Martinistrasse 52, 20246 Hamburg,

Germany

e-mail: amling@uke.de

F. T. Beil $\cdot$ W. Rüther

Department of Orthopedics, University Medical Center

Hamburg-Eppendorf, Martinistrasse 52, 20246 Hamburg, Germany

M. Steiner · A. Ignatius

Institute of Orthopaedic Research and Biomechanics, Center of

Musculoskeletal Research, University of Ulm, 89081 Ulm, Germany
Results The fluoride content of water, blood and urine was significantly elevated in the Kalahari group compared to the control. Surprisingly, a significant decrease in both cortical and trabecular bones was found in sheep chronically exposed to fluoride. Furthermore, osteoid parameters and the degree and heterogeneity of mineralization were increased. The latter findings are reminiscent of those found in osteoporotic patients with treatment-induced fluorosis. Mechanical testing revealed a significant decrease in the bending strength, concurrent with the clinical observation of fragility fractures in sheep within an area of environmental fluoride exposure.

Conclusions Our data suggest that fluoride exposure with concomitant calcium deficit (i) may aggravate bone loss via reductions in mineralized trabecular and cortical bone mass and (ii) can cause fragility fractures and (iii) that the prevalence of skeletal fluorosis especially due to groundwater exposure should be reviewed in many areas of the world as low bone mass alone does not exclude fluorosis.

Keywords Fluoride $\cdot$ Fluorosis $\cdot$ Fragility fracture Osteoporosis

\section{Introduction}

Fluoride is part of the environment, being naturally present in water, rocks and soil throughout the world but with tremendous regional differences explained by tectonic plate movements [1-5]. Fluoride is known to interact with mineralized tissues such as bone and teeth and has the potential to increase bone mass and prevent dental caries. In the latter regard, fluoridation of drinking water to prevent dental caries is 
considered as one of the ten most important public health achievements of the twentieth century [6]. However, the pharmacological use of fluorides did not translate into fracture reduction in patients with osteoporosis despite increases in bone mineral density, which explains the loss of interest in the pharmacological use of fluorides in bone loss syndromes. The discrepancy between a successful increase in bone mass and the lack of fracture reduction is at least in part explained by the action of fluorides in the mineralization process as well as in the remodelling process, where bone quality is compromised and the interaction between osteoblasts and osteoclasts is altered [7-10]. Nevertheless, as environmental exposure to fluorides from various sources (including workplace exposure, environmental pollution and fallout), but especially as daily intake of naturally fluoridated water, is inevitable in many areas and countries throughout the world, it remains a major health issue as it has been suggested that fluorides may paradoxically aggravate osteoporosis and increase osteoporotic fractures [11]. For example, Jacobsen and coworkers reported a significant positive relation between hip fractures and water fluoride concentration within the USA [12], which was corroborated by similar results for the UK reported by Cooper and coworkers [13]. To date, there is no consensus about whether these observations of increased fracture risk really relate to fluoride-induced bone loss, as increased radiographic density, a generally accepted hallmark diagnostic sign of skeletal fluorosis, was not found in the USA or the UK. Here, it is of critical importance to note that in fact, the diagnosis and/or especially exclusion of skeletal fluorosis is in general made on the basis of the presence or absence of osteosclerosis on X-rays, in other words, decreased radiolucency, without further histological confirmation to exclude mineralization defects and bone quality deterioration. However, as the rates of dental fluorosis in the USA have dramatically increased during the last 60 years, with a prevalence of $10 \%$ in 1950, $23 \%$ in 1987 and $41 \%$ in 2004 [14], the US Department of Health and Human Services (DHHS) has announced its recommendation that water fluoridation programmes should lower the levels added (to $0.7 \mathrm{mg} / 1$ [=parts per million (ppm)]) in January 2011 [15]. Thus, there is an awareness of potential clinical problems beyond classical fluorosis that might be of importance not only for people in certain areas of the world with a naturally high fluoride water content due to pyroclastic/geologic activity in the past but also for communities whose water has been and is still been being fluoridated. On the latter basis, we decided to study the skeletal phenotype of sheep from a flock presenting with fragility fractures-which is completely unusual for livestock-known to be chronically exposed to highly naturally fluoridated groundwater in the Kalahari Desert.

In order to study the skeletal phenotype, we decided to use the following devices and methods: After laboratory analyses of drinking water, sheep blood and urine, radiographic images and analyses were undertaken to assess structural changes in femora and iliac crest biopsies. Mineralization changes in the bone following exposure to fluoride needed to be analysed. Histology and histomorphometry are essential to identify cellular differences, and for biomechanical property effects, three-point bending tests were executed, yielding explanations for the fragility fractures occasionally observed in sheep.

\section{Methods}

Animals

We studied aged non-fractured sheep from a flock observationally reported to present with fragility fractures and compared them with sheep of the same breed from a flock without fragility fractures. Therefore, ten Dorper ewes aged 6-7 years were obtained from two farms, five for each group, from two different regions in Namibia. One farm was in a region within the Kalahari Desert and is known for the high fluoride content in the groundwater, whereas the other farm was outside the Kalahari Desert and has normal fluoride levels in the groundwater.

\section{Specimens}

All sheep material used in this study originated from animals that were free range and were grown and fed for meat sale or as a personal meat source for the farmers. Water samples from the livestock drinking wells on both farms were taken and used for further analysis, in particular, with regard to the fluoride content. Blood, urine and bone samples were obtained during the slaughter process prior to sale of the meat on the meat market. Five sheep were derived from a flock of the southwestern Kalahari Desert area where sheep have been reported to present with fragility fractures of long bones without a known significant trauma. The control sheep material from ewes of the same breed as well as water samples were collected at a farm in a different region outside the Kalahari where broken bones have never occurred during the past 10 years.

For direct comparison with changes that occur in humans with fluorosis, we made use of archived iliac crest biopsies from eight patients who had been treated with sodium fluoride regimens for osteoporosis and were supervised at our institution (six females, two 
males; mean age $67.4 \pm 9.4$ years, sodium fluoride average $40 \mathrm{mg}$ /day for $3.7 \pm 1.1$ years). Transiliac crest biopsies from age- and sex-matched individuals without skeletal pathologies obtained from our bone biopsy register were used as human controls.

\section{Water and fluoride analyses}

Groundwater analyses for fluoride were performed by determination of dissolved anions by liquid chromatography of ions using the following standard: DIN EN ISO 10304-1 at the IWW Water Research Institute (Rheinisch-Westfälisches Institut für Wasser, Mühlheim an der Ruhr, Germany). Groundwater was analysed for nitrate $(\mathrm{mg} / \mathrm{l})$, fluoride $(\mathrm{mg} / \mathrm{l})$, chloride $(\mathrm{mg} / \mathrm{l})$, sulphate $(\mathrm{mg} / \mathrm{l})$, sodium $(\mathrm{mg} / \mathrm{l})$, potassium $(\mathrm{mg} / \mathrm{l})$, calcium $(\mathrm{mg} / \mathrm{l})$ and magnesium $(\mathrm{mg} / \mathrm{l})$.

Fluoride in blood and urine was determined potentiometrically using an ion-selective electrode in the laboratories of Medical Laboratory Bremen (Medizinisches Labor Bremen, Bremen, Germany).

Quantitative backscattered electron imaging and energy-dispersive X-ray spectroscopy

The distribution of bone minerals was investigated in the iliac crest specimens from the Dorper ewes and patients described above. The surface of the plasticembedded block specimens was polished and carboncoated for scanning electron microscopy (LEO $435 \mathrm{VP}$, Leo Electron Microscopy Ltd., Cambridge, England) and nondestructive microanalysis with energy-dispersive X-ray analysis (EDX; DX-4, Mahwah, NJ), allowing spatial investigation of the elemental concentrations $[16,17]$. To determine the elemental composition of the bone specimens, EDX was performed in the centre of trabecular nodes with a transverse section $>100 \mu \mathrm{m}$. These regions of interest (ROI) were each $0.025 \mathrm{~mm}^{2}$ in size. Elemental peaks reflecting pronounced calcium $(\mathrm{Ca})$, phosphorus $(\mathrm{P})$, oxygen $(\mathrm{O})$, sodium $(\mathrm{Na})$, magnesium $(\mathrm{Mg})$ and fluorine $(\mathrm{F})$ content in the bone were evaluated in weight percent (Wt\%) via EDX-ZAF software provided by the manufacturer. EDX quantifies the relative contribution of each of the detected elements (within the ROI) to $100 \%$.

Quantitative backscattered electron microscopy was used to assess the degree of mineralization of the specimens (sheep-iliac crest and femoral cortex, humaniliac crest). The application is based on the work of other groups that use quantitative backscattered electron imaging (qBEI) [18-20] and has been reported previously $[21,22]$. The pixel size of $3 \mu \mathrm{m}$ was determined based on the recommendations of Roschger and colleagues [20]. The generated grey values represent the mean calcium content (mean $\mathrm{Ca}-\mathrm{Wt} \%$ ) and the heterogeneity of mineralization (width $\mathrm{Ca}-\mathrm{Wt} \%$ ) of the cross-sectioned cancellous bone.

Contact radiographs, micro-computer tomography and high-resolution peripheral quantitative computer tomography

Iliac crest biopsies and the femora of all sheep were analysed by contact radiography using a Faxitron X-ray cabinet (Faxitron X-ray Corp., Wheeling, IL, USA). For the assessment and 3D visualization of the cortical thickness, porosity, bone volume per tissue volume (BV/TV), trabecular thickness (Tb.Th), trabecular number (Tb.N) and trabecular spacing (Tb.Sp), iliac crest biopsies were scanned $(40 \mathrm{kV} / 114 \mathrm{~mA})$ using a micro-computer tomography $(\mu \mathrm{CT}) 40$ (Scanco Medical AG, Bruettisellen, Switzerland). The scanning process and analysis were executed as previously described [23]. Measurements of femora cortex were performed at midshaft. Further structural assessment and 3D visualization of bone volume (BV/TV), trabecular number (Tb.N), trabecular spacing (Tb.Sp), trabecular thickness (Tb.Th), cortical thickness (Ct.Th) and porosity were performed with a high-resolution peripheral quantitative computed tomography scanner (HRpQCT, Xtreme-CT ${ }^{\circledR}$, Scanco Medical, Bruettisellen, Switzerland). Therefore, the total femora were scanned $(60 \mathrm{kV} /$ $900 \mu \mathrm{A})$ at a resolution of $80 \mu \mathrm{m}$. Additionally, a cross section of each contralateral femur was scanned $(40 \mathrm{kV} / 114 \mathrm{~mA})$ using a $\mu$ CT 40 (Scanco Medical, Bruettisellen, Switzerland) at a resolution of $18 \mu \mathrm{m}$. Subsequently, the generated raw data were manually segmented for analyses with the $\mu \mathrm{CT}$ Evaluation Program V6.0 (Scanco Medical). Finally, the segmented data were imported and displayed in $\mu \mathrm{CT}$ Ray V3.8 for visualization (Scanco Medical).

Histology, histomorphometry, quantitative analysis of canalicular connections and biomechanical testing

Iliac crest bones were fixed in $3.7 \%$ PBS-buffered formaldehyde for 3 days at $4{ }^{\circ} \mathrm{C}$ and transferred into $70 \%$ ethanol. The biopsies were dehydrated in ascending alcohol concentrations and embedded in methylmethacrylate as described previously [24]. Sections of $5 \mu \mathrm{m}$ were cut on a Microtec rotation microtome (Techno-Med, Munich, Germany). Sections were stained with toluidine blue, trichrome Goldner and the van Gieson/von Kossa procedure as described previously [25].

Quantitative histomorphometry was performed on undecalcified iliac crest sections of 5- $\mu \mathrm{m}$ thickness stained as mentioned above. Analysis of osteoid volume (OV/BV), osteoid surface (OS/BS), osteoid thickness (O.Th) as well as the determination of osteoblast number (N.Ob/B.Pm), osteoclast number (N.Oc/B.Pm) and surface indices (Ob.S/BS and 
Oc.S/BS) were carried out according to standardized protocols using OsteoMeasure histomorphometry system (OsteoMetrics, Atlanta, GA, USA) and BIOQUANT (BIOQUANT Image Analysis, Inc., Nashville, TN, USA) according to the American Society for Bone and Mineral Research (ASBMR) standards [26]. Furthermore, buried osteoid was characterized beyond standard ASBMR nomenclature (number of cases/total cases). Polarized images and analyses of histological sections were executed for qualitative assessment of the osseous maturation and mineral deposition (e.g. distinction between woven and lamellar bone) as previously described [27].

To examine the canalicular network, polished sheep femur bone samples were exposed to acid etching following earlier recommendations by Kubek [17] and Milovanovic [17, 28]. After acid etching, the surface was coated with gold (Au) using a sputter coater (Cressington 108, Cressington Sc. Instr. Ltd., Watford, UK). Thereafter, the specimens were analysed in a scanning electron microscope (LEO 435 VP; LEO Electron Microscopy Ltd., Cambridge, England). Measurement of the number of canaliculi per osteocyte lacuna (N.Ot.Ca/Ot.Lc) was done through digital image analysis as previously described [17]. The data for all lacunae were averaged for each specimen.

The biomechanical properties of the cortical bone of the sheep femora were evaluated using three-point bending tests. We used a standard materials testing machine (1454, Zwick $\mathrm{GmbH}$, Ulm, Germany) equipped with a $10-\mathrm{KN}$ load cell (Hottinger Baldwin Messtechnik GmbH, Leonberg, Germany) to perform the three-point bending tests. The deflection of the femoral midshaft was measured using an external measuring device (MT25; Heidenhein, Traunreut, Germany). The surrounding soft tissues were removed from the femora, and proximal and distal ends were embedded using Technovit 3040 (Heraeus Kulzer GmbH, Wehrheim, Germany) into aluminium cylinders, which were placed on two supports spaced $l=140-\mathrm{mm}$ apart. Each femur was then loaded with an indenter on the medial bone surface at a speed of $1 \mathrm{~mm} /$ min, and the deflection of the lateral bone surface was measured using an external measuring device. The subsidence of the specimen was assessed using two loading cycles with a maximum load of $200 \mathrm{~N}$; in the third cycle, the femora were loaded until failure. The bending stiffness $S_{\mathrm{b}}$ and the ultimate bending load $F_{\text {max }}$ of each specimen were assessed using force-deflection curves. Hence, the bending stiffness was calculated based on the slope $k_{\mathrm{b}}$ of the force-deflection curve using the following equation: $S_{b}=k_{b} \cdot \frac{l^{3}}{48}$. Consequently, the apparent Young's modulus $E_{\text {app }}$ and the bending strength $\sigma_{\max }$ of each specimen were calculated using the equations $E_{a p p}=\frac{S_{b}}{I_{y}}$ and $\sigma_{\max }=\frac{F_{\max } \cdot l}{4} \cdot \frac{a}{I_{y}}$. The outer radius in the lateral direction $\alpha$ of the diaphyseal cortex and the second moment of area with reference to the anterior-posterior axis $I_{y}$ were defined from HR-pQCT slices as described previously [29].
Statistical analysis

Statistical analysis was performed using the unpaired one-way Student's $t$ test. Levels of significance were defined as significant when $p \leq 0.05$ and/or $p \leq 0.01$.

\section{Results}

Water and fluoride analyses

The water analyses confirmed that the farm in the southwestern part of the Kalahari Desert had a significantly higher fluoride content than the farm in the central part of Namibia, at $9.8 \pm 0.3$ vs. $0.64 \pm 0.32 \mathrm{mg} / \mathrm{l}$ of fluoride in the water, respectively (Fig. 1a-c). Further groundwater analyses revealed additional salt differences, specifically, higher sodium (3,410.0 vs. $114.0 \mathrm{mg} / \mathrm{l})$, nitrate (165.0 vs. $87.6 \mathrm{mg} / \mathrm{l})$, chloride (1,680.0 vs. $27.1 \mathrm{mg} / \mathrm{l})$ and sulphate (1,680.0 vs. $27.9 \mathrm{mg} / \mathrm{l})$; a similar potassium content (13.7 vs. $12.9 \mathrm{mg} / \mathrm{l})$; and lower calcium ( $<5.0$ vs. $17.6 \mathrm{mg} / \mathrm{l})$ and magnesium $(<5.0$ vs. $31.3 \mathrm{mg} / \mathrm{l})$ in the Kalahari Desert area.

The biological intestinal uptake of fluoridated water was confirmed by blood and urine analyses. A total of $165.25 \pm$ $74.4 \mu \mathrm{g} / \mathrm{l}$ fluoride in blood and $1.17 \pm 0.7 \mu \mathrm{g} / \mathrm{l}$ fluoride in urine were registered in the fluoride sheep group. These values were significantly higher than those in the control sheep, in which fluoride levels in both blood and urine were below the detection limit (Fig. 1c).

\section{Energy-dispersive X-ray spectroscopy}

In sheep exposed to highly fluoridated water, fluoride is incorporated into bone, as demonstrated by a fluorine peak in elemental analysis of the iliac crest (Fig. 1f), whereas no such peak occurred in samples from the control group (Fig. 1d). Figure $1 \mathrm{~h}$ indicates a significant quantitative difference in the weight percent levels for fluorine of the elements detected in the iliac crest bone of the sheep. Of note, similar fluorine incorporation was detected in human bone samples with fluoride treatment-associated osteopathy and intact human bone samples (Fig. 1e, g and i).

Radiographs, $\mu \mathrm{CT}$, HRpQCT, biomechanical testing

The contact radiographs and the Xtreme-CT 3D reconstructions of the femoral diaphyses (Fig. 2) showed reduced cortical thickness in the fluoride group (Fig. 2b) in comparison to control animals (Fig. 2a). Quantitative analysis of the structural parameters was validated by $\mu \mathrm{CT}$ scans of femur cross sections, confirming the surprising visual impression of a significant decrease in cortical thickness and a significant 
Fig. 1 Local situation in Namibia showing groundwater wells with wind- and solar-powered pumps (a) and Dorper sheep from the southwestern Kalahari Desert (b). Fluoride content of groundwater, blood and urine samples from the sheep (c). EDX peaks indicating fluoride elevation in sheep (f) and in human (g) samples compared to their controls, respectively (d,

e). Bar graphs demonstrating fluoride in weight percent in sheep (h) and human (i) specimens. (significance at ${ }^{*} p<0.05$ or at $\left.{ }^{* *} p<0.01\right)$

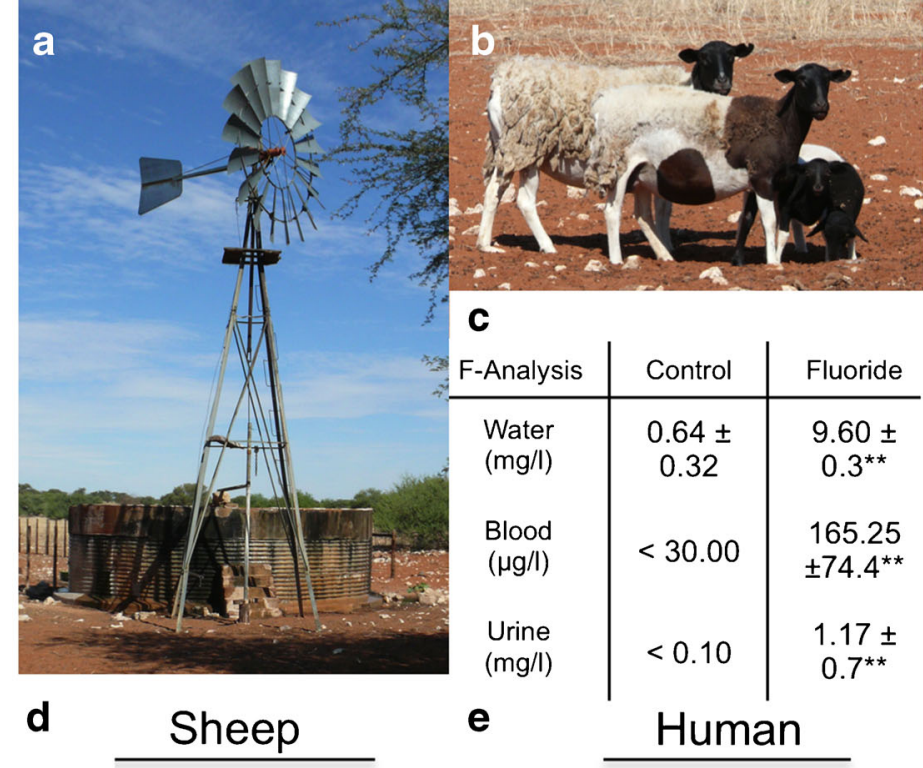

EDX Control
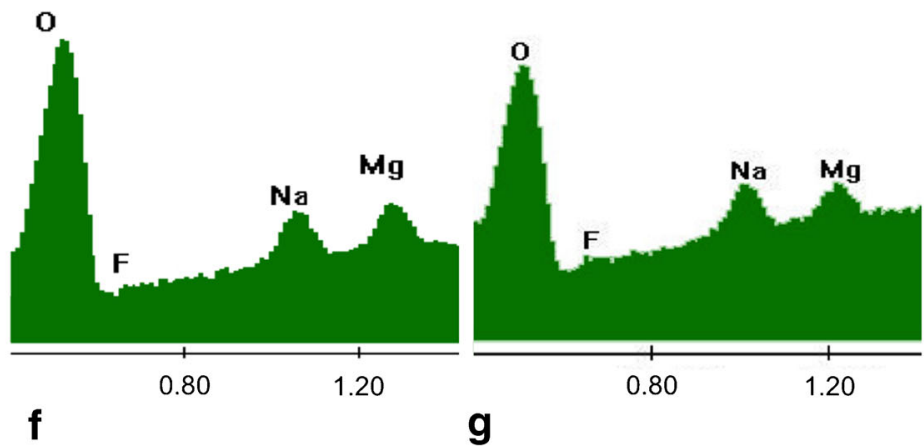

f

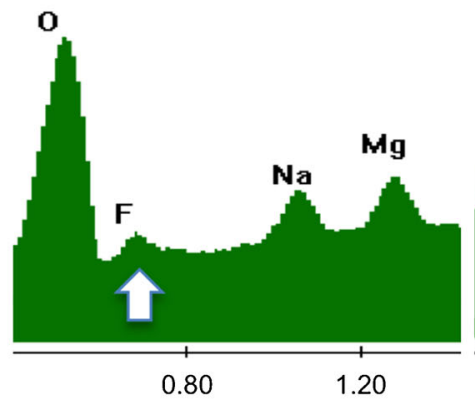

h

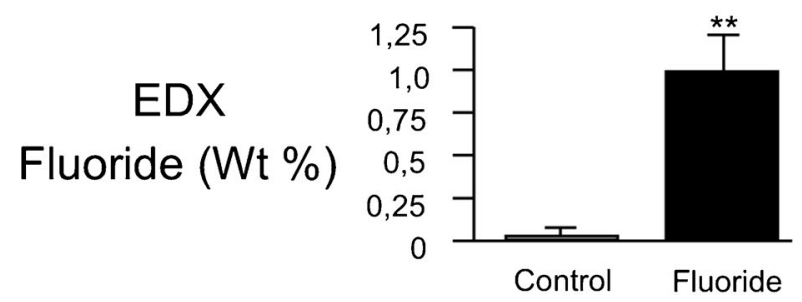

Fluoride (Wt \%)

EDX Fluoride

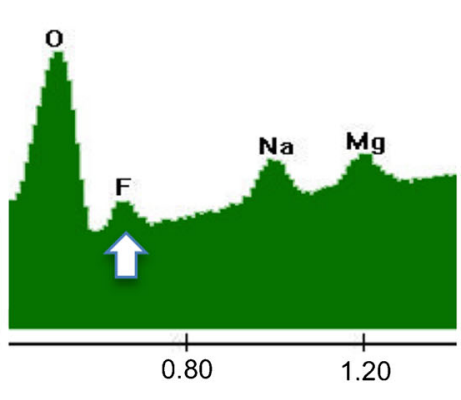

i

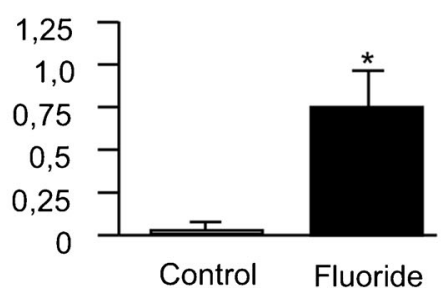

increase in both cortical porosity for the fluoride sheep (Fig. 2c).

Contact radiographs of the iliac crest bone samples from fluoride-drinking sheep demonstrated an unexpected increase in radiolucency (Fig. 2e) compared to controls (Fig. 2d); the latter was confirmed by independent $\mu \mathrm{CT}$ evaluation (Fig. 2f). CT-based morphometry showed a significant decrease in bone volume fraction in the fluoride group (BV/TV 25.6 $\pm 5.0 \%$ ) compared to the controls (BV/TV 35.0 $\pm 5.5 \%$ ). Changes of a similar magnitude were observed for trabecular thickness, 
Fig. 2 Analyses of trabecular and cortical bone of sheep femur $(\mathbf{a}-\mathbf{c})$ and iliac crest $(\mathbf{d}-\mathbf{f})$ biopsies according to the nomenclature proposed by Parfitt and colleagues [26]. Diaphysis contact X-rays of the midshaft region and Xtreme-CT midshaft reconstruction images (a and $\mathbf{b}$ ) show in the table section the calculated effects of cross sections of femora at midshaft scanned with $\mu \mathrm{CT}$ (c). Analyses of the iliac crest (f) performed after taking contact X-rays (upper part of $\mathbf{d}$ and $\mathbf{e}$ ). Reconstructed iliac crest samples for control (d) and fluoride-exposed sheep (e) are pictured in the lower part. (significance at $* p<0.05$ or $* * p<0.01$ )

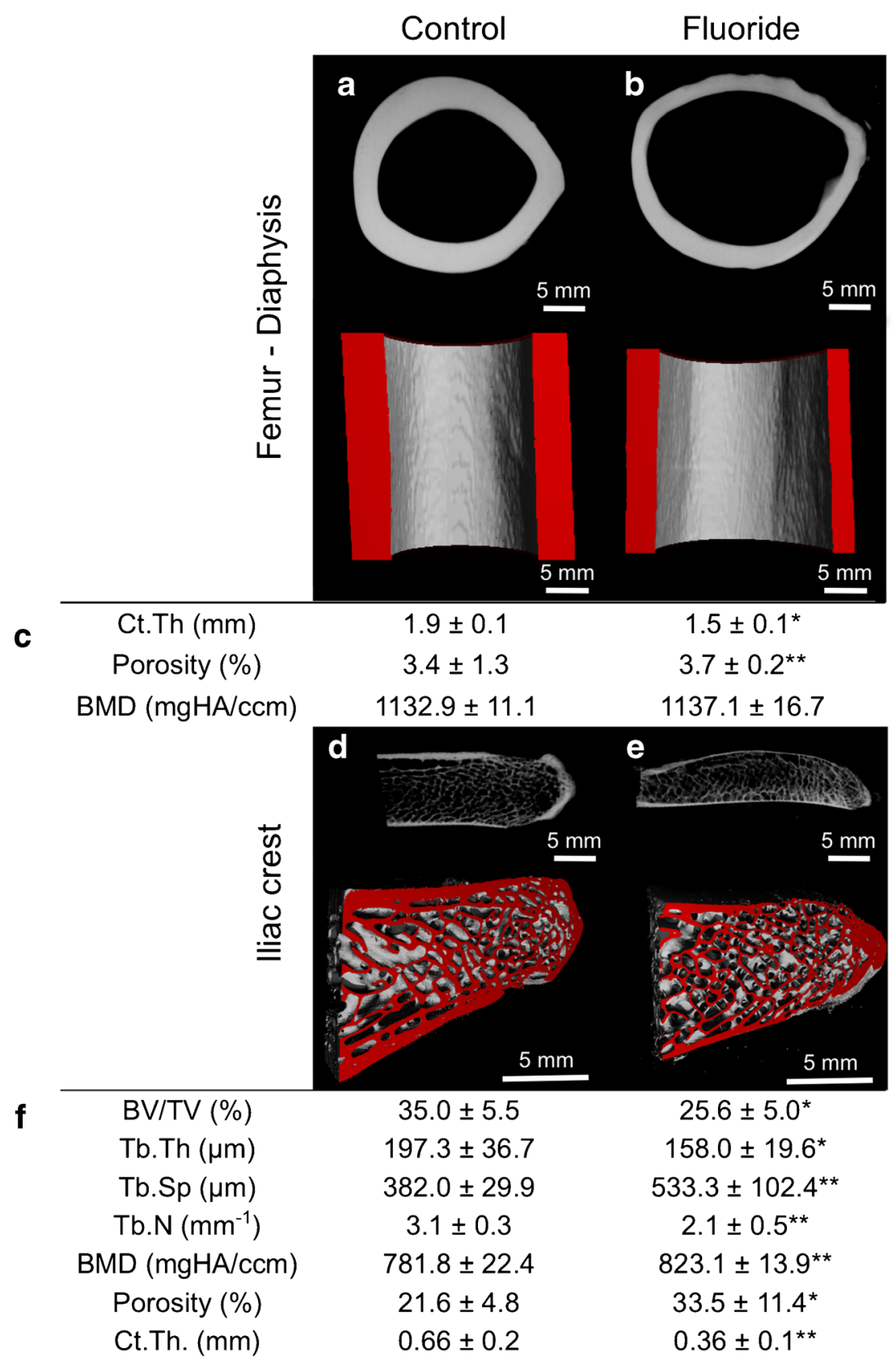

trabecular spacing, trabecular number, BMD, porosity and cortical thickness (Fig. 2f).

To address whether these structural differences are of biomechanical relevance and thus might explain the observation of fragility fractures in the affected sheep, mechanical testing was performed. Indeed, the biomechanical properties of the sheep femora were negatively affected by the lifelong intake of highly fluoridated water. The ultimate bending load $\left(\mathrm{F}_{\max }\right)$ of $1,462 \pm 341 \mathrm{~N}$ in the fluoride group was significantly decreased than that of the control group, at 1,790 \pm 203 N. Furthermore, the bending strength $\left(\sigma_{\max }\right)$ was significantly decreased in the fluoride group with earlier fracture, at $97 \pm$
$15 \mathrm{MPa}$ (fluoride group) and 116 $\pm 11 \mathrm{MPa}$ (control samples) (Table 1).

Histology and histomorphometry

At the bone tissue level, histomorphometric analysis revealed a significant increase in osteoid volume $(\mathrm{OV} / \mathrm{BV})$ and osteoid surface $(\mathrm{OS} / \mathrm{BS})$ in the iliac crest biopsies from the Kalahari group, at $6.03 \pm 1.48 \%$ and $51.28 \pm 6.01 \%$, respectively (Fig. 3). The osteoid thickness (O.Th) increased from $8.14 \pm$ $2.51 \mu \mathrm{m}$ in the control group to $12.57 \pm 3.01 \mu \mathrm{m}$ in the fluoride group of sheep. Buried osteoid was detected in all fluoride 
Table 1 Biomechanical testing of sheep femora

\begin{tabular}{|c|c|c|c|c|c|c|}
\hline & $I_{\mathrm{y}}\left(\mathrm{mm}^{4}\right)$ & $\alpha(\mathrm{mm})$ & $S_{\mathrm{b}}\left(\mathrm{Nm}^{2}\right)$ & $F_{\max }(\mathrm{N})$ & $E_{\text {app }}(\mathrm{MPa})$ & $\sigma_{\max }(\mathrm{MPa})$ \\
\hline Control & $5,352 \pm 1,253$ & $9.8 \pm 0.8$ & $96 \pm 16$ & $1,790 \pm 203$ & $18,201 \pm 1,932$ & $116 \pm 11$ \\
\hline Fluoride & $5,226 \pm 638$ & $10.0 \pm 0.4$ & $99 \pm 14$ & $1,462 \pm 341^{*}$ & $19,000 \pm 1,390$ & $97 \pm 15^{*}$ \\
\hline
\end{tabular}

The bending stiffness $S_{\mathrm{b}}$ and ultimate bending load $F_{\max }$ of the sheep femora were determined using a three-point bending test. The apparent Young's modulus $E_{\text {app }}$ and the bending strength $\sigma_{\max }$ were computed using the second moment of inertia $I_{\mathrm{y}}$ and the outer diameter of the cortex in the lateral direction $\alpha$ at the femora diaphysis

${ }^{*} p<0.05 ;{ }^{*} p<0.01$

group samples (Fig. 3b, d), while it was absent from controls (Fig. 3a, c).

Next, we put these findings in sheep into clinical perspective and used an approach to translate the bone tissue changes observed in sheep to human patients (Fig. 4). In fact, the histopathological findings were absolutely similar in sheep and man. Patients diagnosed with fluorosis (Fig. 4b, d) while receiving fluoride treatment for osteoporosis demonstrated the same significant differences in osteoid volume (7.94 \pm 3.56 vs. $<2.00 \%$ ), osteoid surface $(25.92 \pm 7.64$ vs. $<21.00 \%)$ and osteoid thickness $(13.5 \pm 3.25$ vs. $<12.00 \mu \mathrm{m})$ as the sheep from the Kalahari Desert (Fig. 3 and 4). Most importantly,
Fig. 3 Iliac crest biopsies of sheep samples were obtained, and histomorphometric analysis was performed starting with bone volume per tissue volume $(B V /$ $T V$ ). In addition to osteoid volume per bone volume $(O V / B V)$, osteoid surface per bone surface $(O S / B S)$, osteoid thickness $(O . T h$.$) and the number of buried$ osteoid cases per total cases, the number of osteocytes (N.Oc) B.Pm) and osteoblasts (N.Ob/ B.Pm) per bone perimeter, osteoclast surface $(O c . S / B S)$ and osteoblast surface $(O b . S / B S)$ per bone surface were evaluated. The histological slides were stained with a Masson-Goldner dye (a and $\mathbf{b}$ ) and toluidine blue dye for polarized light analysis (c and d) (significance at $* p<0.05$ or at $* * p<0.01$ )
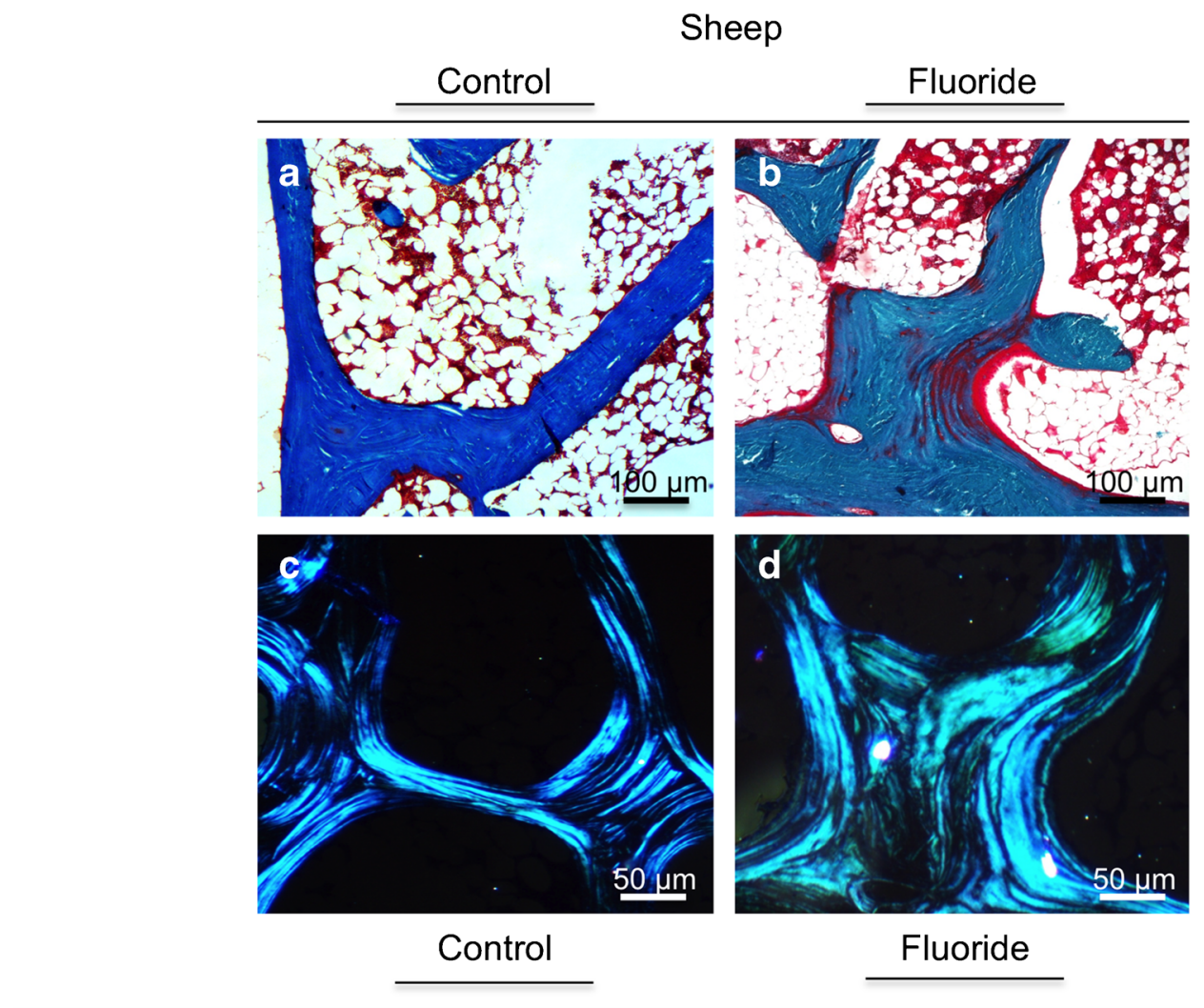

Fluoride

\begin{tabular}{ccc}
\hline BV/TV $(\%)$ & $30.8 \pm 2.3$ & $22.4 \pm 1.1^{* \star}$ \\
OV/BV $(\%)$ & $1.14 \pm 0.50$ & $6.03 \pm 1.48^{\star *}$ \\
OS/BS $(\%)$ & $20.95 \pm 5.04$ & $51.28 \pm 6.01^{\star \star}$ \\
O.Th $(\mu \mathrm{m})$ & $8.14 \pm 2.51$ & $12.57 \pm 3.01^{*}$ \\
Buried Osteoid (x/cases) & $0 / 5$ & $5 / 5^{\star \star}$ \\
N.Oc/B.Pm $\left(\mathrm{mm}^{-1}\right)$ & $1.1 \pm 0.2$ & $1.9 \pm 0.5^{\star}$ \\
N.Ob/B.Pm $\left(\mathrm{mm}^{-1}\right)$ & $8.1 \pm 1.2$ & $14.3 \pm 2.1^{\star}$ \\
Oc.S/BS (\%) & $1.6 \pm 0.2$ & $2.4 \pm 0.6^{\star}$ \\
Ob.S/BS (\%) & $12.1 \pm 3.5$ & $22.6 \pm 3.9^{\star \star}$
\end{tabular}


Fig. 4 Histomorphometric analyses of iliac crest biopsies of human specimens. Osteoid volume per bone volume $(\mathrm{OV} /$ $B V$ ), osteoid surface per bone surface $(O S / B S)$, osteoid thickness $(O . T h)$ and the number of buried osteoid cases per total cases were measured. Section sign indicates reference values for healthy human controls derived from a previous study by Priemel and colleagues [50]. The histological slides were stained with a Masson-Goldner dye promoting non-mineralized tissue and buried osteoid (a and $\mathbf{b}$ ). Polarized histological images were obtained from toluidine blue stained slides (c and d) (significance at $* p<0.05$ or at $* * p<0.01)$

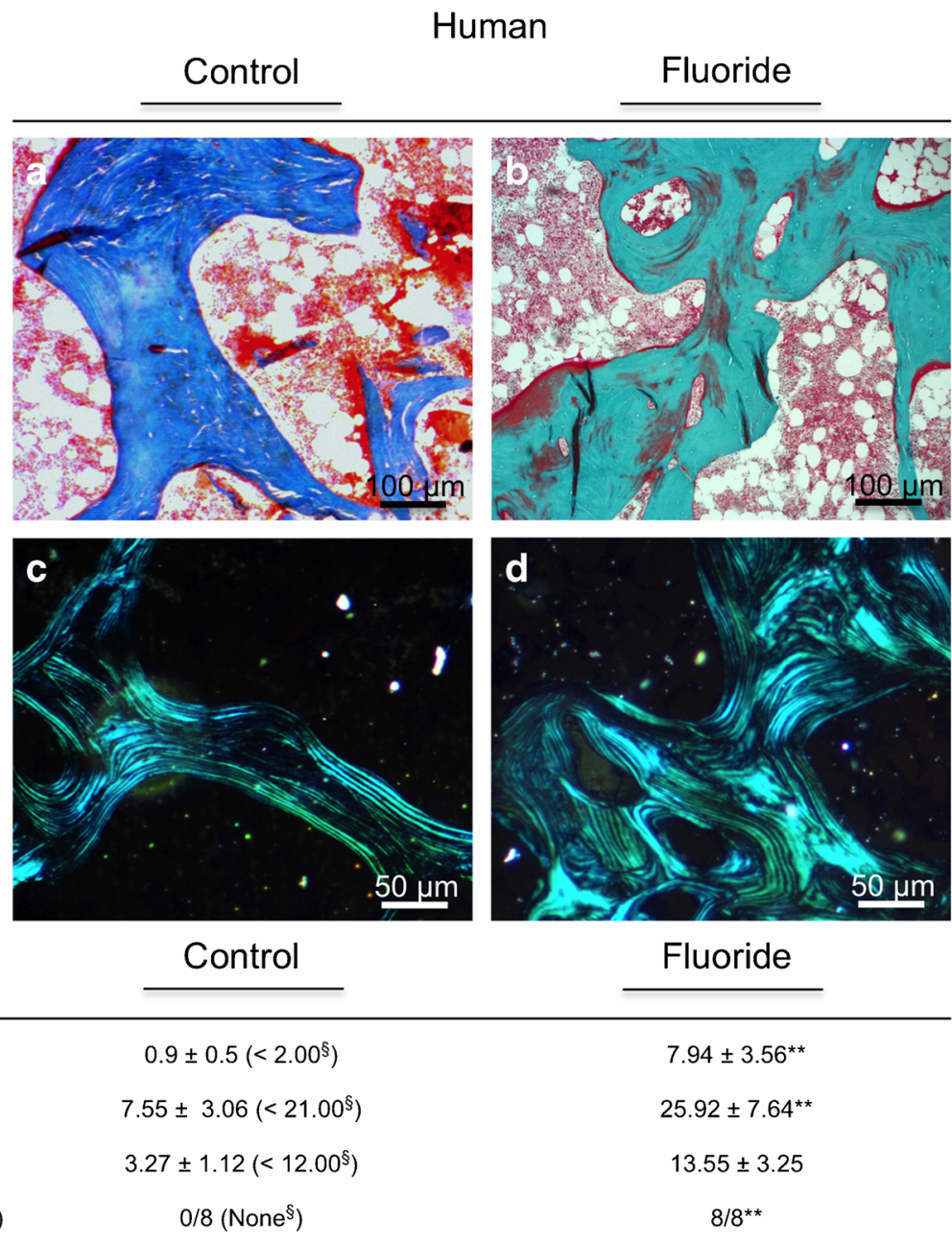

buried osteoid was absent from the control group (Fig. 4a, c), whereas buried osteoid was found in all cases receiving fluoride treatment (Fig. 4), exactly mirroring the findings in sheep (Fig. 3).

To further characterize the bone remodelling state of the sheep, we performed histomorphometry of the relevant major cell populations on iliac crest biopsies. This analysis revealed a significant increase in all parameters - osteoblast number and surface as well as osteoclast number and surface - in the fluoride group in comparison to the control (Fig. 3).

\section{Quantitative backscattered imaging}

The results from qBEI in Dorper sheep and human specimens (trabecular bone of iliac crests) demonstrate that the mean calcium weight percentages were significantly increased in the fluoride-exposed samples for both sheep and man when compared with their control groups (Fig. 5a, b, e, f). The heterogeneity of the bone tissue - the calcium width-differed in both sheep and human iliac crest biopsies (Fig. 5c, g). In addition, the calcium peak was significantly increased in the fluoride samples of both species (Fig. 5d, h). These results were confirmed by qBEI analysis of the cortical bone of sheep femora (Fig. 5j-1).

Further analyses of qBEI images of the sheep femora showed a significant increase in secondary osteons (N.H.Ca./B.Ar. = number of haversian canals per bone area) in the fluoride group $(0.525 \pm 0.374$ vs. $0.121 \pm 0.142$ in the control samples) (Fig. 6a, b). The number of osteocyte canaliculi per osteocyte lacunae (N.Ot.Ca./Ot.Lc) in the fluoride group differed significantly to that of control samples $(14.1 \pm$ 2.7 vs. $21.5 \pm 3.1$ ). In contrast, the lacunar area (Lc.Ar.) and the number of osteocytes (N.Ot $/ \mathrm{mm}^{2}$ ) did not differ significantly between both groups (Fig. 6c, d).

\section{Discussion}

In many areas throughout the world, natural exposure to fluoride - not only through groundwater but also through occupational exposure [30] or fallout [31] - is excessive and thus might cause health problems in the community 
Sheep Iliac Crest

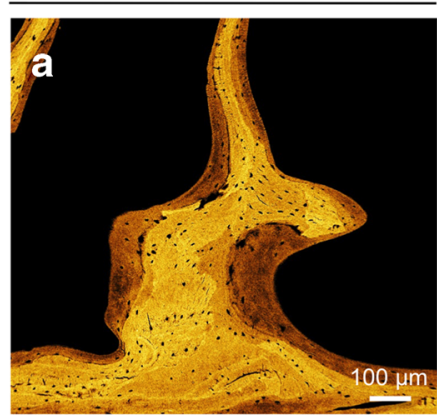

b

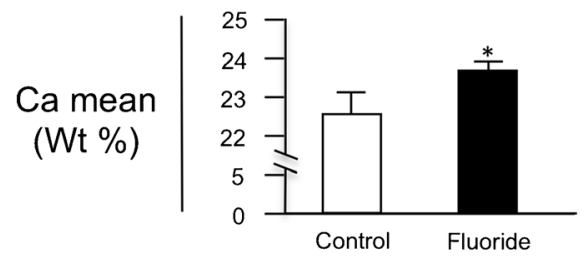

c

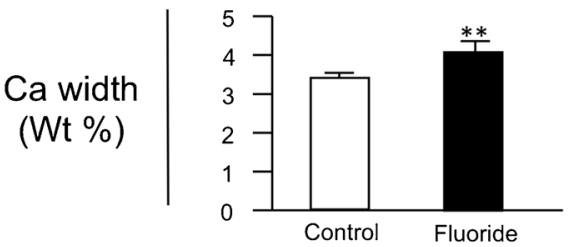

d

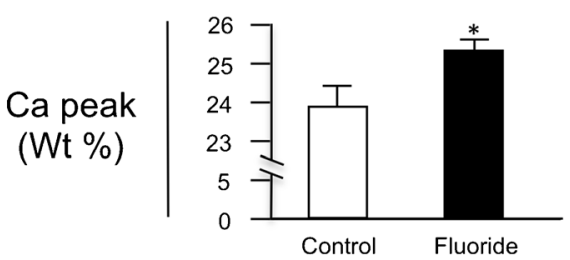

Human Iliac Crest

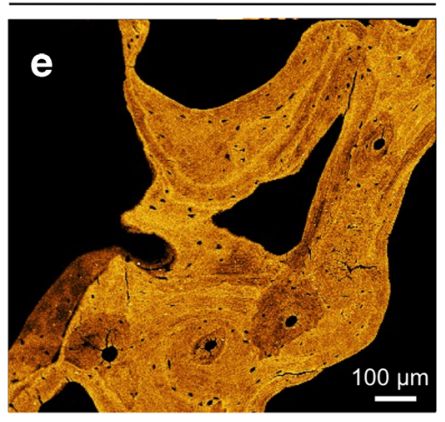

f

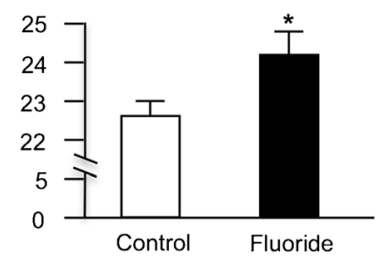

g

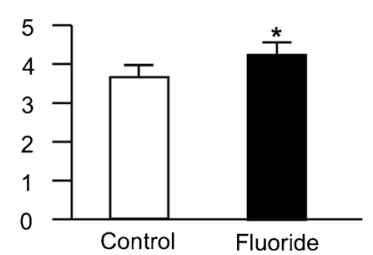

h

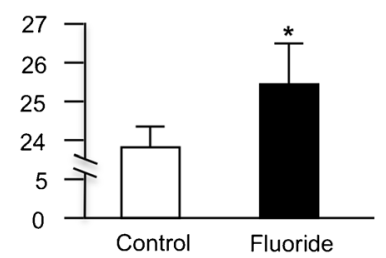

Sheep Femur

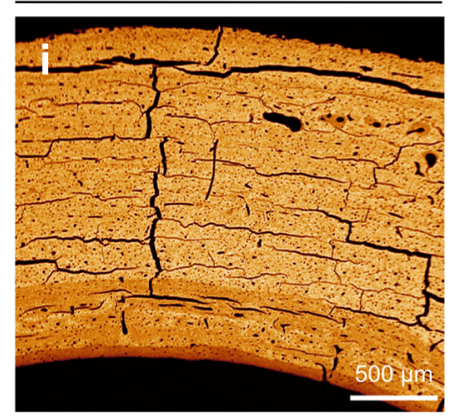

j
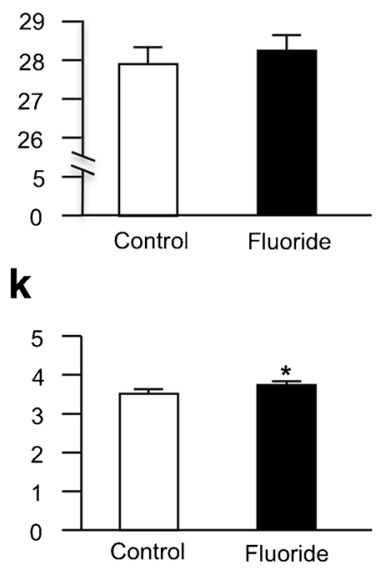

I

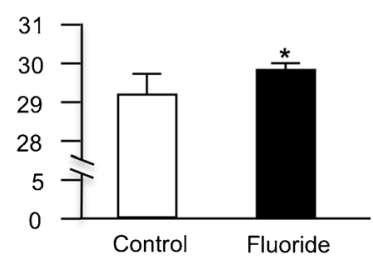

Fig. 5 Quantitative backscattered electron imaging (qBEI) analysis results and images. The images include a coloured qBEI image of bone mineralization for fluoride-exposed sheep (a iliac crest, $\mathbf{i}$ femur) and for human patients who had been treated with fluoride for osteoporosis (e). Mineralization mean (b), width (c) and peak (d) for sheep are also shown.
Human sample results are shown in the form of bar graphs $(\mathbf{f}, \mathbf{g}, \mathbf{h})$ and demonstrate significant differences between fluoride and control samples. The results for sheep femora are also shown $(\mathbf{j}, \mathbf{k}, \mathbf{l})$. (significance at $* p<0.05$ or at $* * p<0.01$ ) inhabitants exposed [3]. Nutritional fluoride intake should also be taken into account, as even excessive tea drinking can lead in single cases to fluoride intoxication [32, 33]. Just focusing on drinking water, it has been reported that even in developed countries, far more than 7 million people are regularly exposed to water with a fluoride content of 2 to $10 \mathrm{mg} / \mathrm{l}$, thus exceeding the upper tolerable limit for drinking water, which was defined as $1.5 \mathrm{mg} / \mathrm{l}$ fluoride by the WHO in 2004, and as adopted by the EU within the guideline $98 / 83 / \mathrm{EG}$ and the German guidelines (Trinkwasserverordnung 2001). The most commonly described health problems are dental fluorosis or skeletal fluorosis, and these have been found on several continents [2, 3, 34-36]. Symptoms of skeletal fluorosis and radiological signs vary and are reported to occur with chronic fluoride incorporation starting at $4 \mathrm{mg} / \mathrm{l}$ [3]; the latter presumed threshold is based on the fact that in epidemiological studies conducted in the USA, no radiographic increases in bone density were detected in persons drinking water containing less than $4 \mathrm{mg} / \mathrm{l}$ fluoride [37-40].

According to Kaminsky and coworkers' [41] review published in 1990, skeletal fluorosis is defined as follows and has several stages: "two preclinical asymptomatic stages characterized by slight radiographically detectable increases in bone mass; and early symptomatic stage characterized by sporadic pain and stiffness of joints and osteosclerosis of the pelvis and the vertebral column; a second clinical phase associated with chronic joint pain, arthritic symptoms, slight calcification of ligaments, sometimes accompanied by osteoporosis of long 
Fig. 6 Quantitative backscattered electron imaging (qBEI) (a, b) and scanning electron microscopy (SEM) imaging $(\mathbf{c}, \mathbf{d})$ of sheep femora and quantitative analysis of canalicular connections. The number of secondary haversian canals per bone area $\left(\mathrm{mm}^{2}\right)$ (N.H.Ca./B.Ar), number of osteocytes per bone area (N.Ot/ $\left.\mathrm{mm}^{2}\right)$, lacunar area per micrometre $\left(L c . A r .\left(\mu \mathrm{m}^{2}\right)\right)$ and the number of osteocyte canaliculi per osteocyte lacunae (N.Ot.Ca./Ot.Lc.) were evaluated. Data are shown in the table below the images (significance at $* p<0.05$ or at $* * p<0.01)$

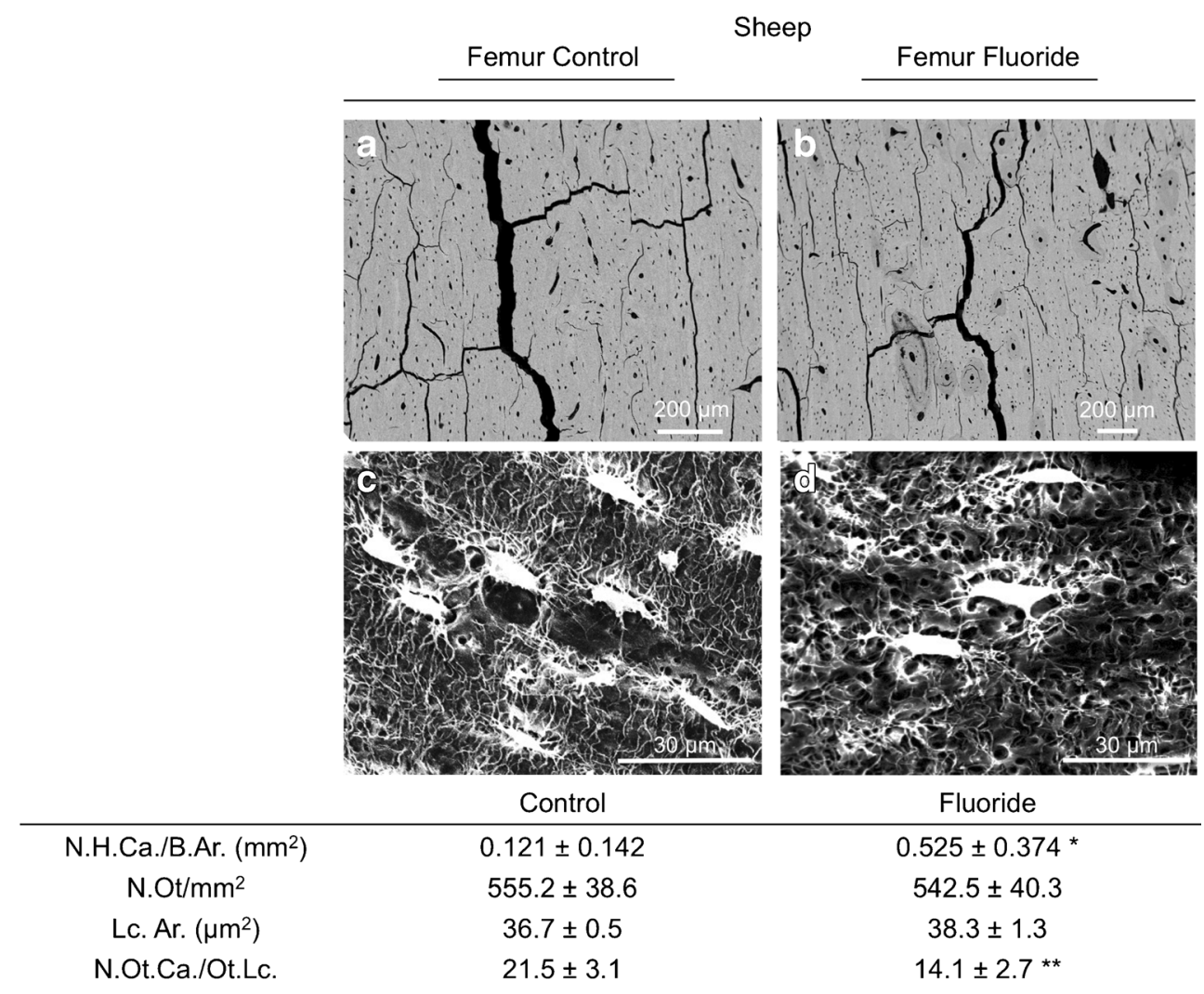

bones; crippling skeletal fluorosis characterized by marked limitation of joint movements, considerable calcification of ligaments, crippling deformities of the spine and major joints, muscle wasting, and neurological defects associated with compression of the spinal cord'.

Here, however, it is of paramount importance to realize that the diagnosis of fluorosis is based on radiologic findings only, the hallmark diagnostic sign being increased bone densityeven if decreased trabecular bone density, at least in long bones, has been reported to occur in one stage of fluorosis [41]! The results of the study presented here challenge this narrow definition and are therefore of broad relevance in terms not only of individual but also of population-based skeletal health issues. In fact, the histologic proof that fluorosis may be associated with and/or even cause low bone mass and decreased bone density challenges the presumed threshold of $4 \mathrm{mg} / \mathrm{l}$ and calls for a review of regional differences in fracture risk, as the exclusion of fluorosis as a potential cause based on previous radiographic analysis of bone density is no longer valid.

Indeed, a study by Sowers and coworkers reported a statistically significant higher incidence of bone fractures in older women (55 to 80 years) but not in younger women (20 to 35 years old) exposed to $4 \mathrm{mg} / \mathrm{l}$ of fluoride in their water versus those exposed to $1 \mathrm{mg} / 1$ [40]. This apparent inconsistency points to - and is best explained by - an associated calcium deficiency as a confounding factor to fluoride action on bone, as enteral calcium absorption is often compromised in older but not in younger women by proton pump inhibitors (PPI) or ageing-associated hypochlorhydria [42], being prevalent in some $20-30 \%$ of people above 60 years of age. In fact, there is evidence that the adverse effects of fluoride are aggravated by a lack of calcium in the diet [43, 44]. And indeed, the sheep from the Kalahari Desert were exposed to water that was high in fluoride and low in calcium and magnesium. The combination may explain the observed unexpected low bone mass phenotype.

It remains to be determined whether the deterioration in bone quality under these conditions is similar to that found in human fluorosis. According to EDX, similar fluoride incorporation was detected in human and sheep bone samples. Analysis employing qBEI revealed that the mean calcium weight percentages were significantly higher in the fluorideexposed samples for both sheep and man and that the heterogeneity of the bone tissue - measured as calcium widthdiffered in both sheep and human iliac crest biopsies, respectively. This trend was also reported by Roschger and colleagues, who showed increased heterogeneity of bone mineralization in a human case of osteoporosis under sodium fluoride treatment [20]. They demonstrated the mineralization spectra to be broader and shifted under fluoride exposure. Similar mineralization changes demonstrating the replacement of old (normal) bone by new (differently mineralized) bone were observed by Fratzl and colleagues [10]. The latter 
data demonstrate that the sheep from the Kalahari indeed present with skeletal fluorosis and thus qualify as a large animal model of fluorosis presenting with low bone mass and compromised bone quality. As expected, mechanical testing of long bones confirmed the reduced mechanical stability, making the bones prone to fracture, thereby revealing chronic environmental fluoride exposure as the cause of fragility fractures reported in livestock from the Kalahari Desert.

"While the stimulatory effects of fluoride on bone formation have been well described, its effect on bone resorption is less well understood45" [45]. There is evidence that fluoride might inhibit bone resorption, while there is also evidence that it might increase bone resorption, especially at high doses. However, so far, there was consensus that the increase of bone formation is an order of magnitude higher than the increase in bone resorption, explaining the net increase of bone mass expected to occur in skeletal fluorosis [46].

We report that in the presence of calcium deficiency, the net effect of fluoride exposure appears to result in low bone mass (osteopenia). Whether this is the result of reduced bone formation at the tissue level (i.e. a reduction in bone formation with accumulation of osteoid that fails to mineralise) or is the result of persisting high remodelling with each remodelling unit depositing less bone than each removed, remains uncertain.

This plausible explanation for the unusual and unexpected low bone mass fluorosis phenotype is corroborated by the finding of Riggs in osteoporotic patients with sodium fluoride treatment, that "[S]upplementary calcium...prevents the increase in bone resorption which occurs when sodium fluoride is given alone [47]."

Therefore, in terms of environmental fluorosis, an increase of bone mineral density should not be expected in areas with either (i) low dietary calcium intake or (ii) in areas with vitamin D deficiency or (iii) in cohorts with high prevalence of PPI users, the latter two conditions causing diminished enteral calcium uptake.

Our data is consistent with the presence of fluorosis with low bone mass in the studied animals by (i) demonstration of fluoride incorporation in bone by EDX, (ii) demonstration of a mosaic pattern and mottled bone by polarized light microscopy (Fig. 3d), (iii) demonstration of increased calcium width by qBEI, (iv) demonstration of stimulated osteoblast activity and increased numbers of secondary haversian channels by quantitative backscattered electron microscopy, (v) demonstration of the absence of filled haversian systems typically seen in sheep with osteomalacia [48] and documentation of a moderate increase in osteoid thickness mirroring the osteoid thickness found in human fluorosis but being in absolute contrast to the massive increase in osteoid thickness described in sheep with osteomalacia [48] and (vi) demonstration of dental fluorosis in all sheep and of the human population living in the area (unpublished data).
This study does, however, have certain limitations. First, we studied free range Dorper sheep, and therefore, these animals were not fluorochrome-labelled prior to sacrifice. This precludes the opportunity for dynamic histomorphometric assessment in addition to the static histomorphometric analysis we did perform. However, we believe that the combined analysis employing radiographs, $\mu \mathrm{CT}, \mathrm{HRpQCT}$, quantitative backscattered imaging as well as histology, static histomorphometry, energy-dispersive Xray spectroscopy and biomechanical testing, respectively, provides a thorough qualitative and quantitative characterization of the effects of chronic fluoride exposure on bone. Second, we focused on characterizing the skeletal differences in 6- to 7 -year-old sheep with and without environmental fluoride exposure and did not analyse sheep of different ages. Therefore, we cannot report on the dynamic development of the fluoride-induced skeletal phenotype during the life cycle of the sheep. Given the results presented here, the latter information would be of interest; however, we believe that this is beyond the scope of the present study. Third, as the sheep in the Kalahari Desert were exposed to groundwater rich in fluoride, but poor in calcium and magnesium, and thus with altered salt content not restricted to fluoride, the observed skeletal phenotype is presumably the result of the combination of chronic fluoride exposure with concomitant calcium deficiency. However, the in vivo proof required to firmly establish this assumption cannot be addressed with free range sheep but requires an experimental setting with sheep housed in metabolic cages, allowing control of nutritional input and balance [49]. Fourth, structural changes in histomorphometric analyses of human fluorosis cases should be interpreted carefully as the underlying osteoporotic disease will inevitably have had a negative influence on the skeleton prior to sodium fluoride therapy.

In summary, we have shown that chronic environmental fluoride exposure (i) may lead to skeletal fluorosis that presents with increased radiolucency due to a reduction in trabecular and cortical bone, (ii) can cause fragility fractures, at least in sheep, at similar fluoride concentrations to those to which many people worldwide are regularly exposed, (iii) is extremely critical when combined with a concurrent calcium deficit and (iv) that the prevalence of human skeletal fluorosis especially due to groundwater exposure should be reviewed in many areas of the world as low bone mass and absence of osteosclerosis on X-rays does not exclude the diagnosis of skeletal fluorosis.

Acknowledgments We thank Marion Dietzmann and Mona Neven for their expertise in the histological processing of the bone samples as well as Tim Rolvien for his assistance with the acid etching process. We also thank Dr. Ulrich Borchers and Dr. Achim Rübel for their help with groundwater analyses at the IWW Water Research Institute and Dr. Helmut Dietrich Köster and Dr. Peter Heitland at Medizinisches Labor Bremen for their help in the analysis of blood and urine. Finally, we thank the farmers in the 
Kalahari area for their unrestricted support and friendly cooperation. This work was supported by the German Research Foundation (DFG) within the framework of the Transregional DFG Research Group 793 to PP, AI, TS and MA. RO, MJKS and FTB are fellows of the German Research Foundation (DFG AM 103/13-1 and DFG AM 103/14-1).

\section{Conflicts of interest None.}

Open Access This article is distributed under the terms of the Creative Commons Attribution Noncommercial License which permits any noncommercial use, distribution, and reproduction in any medium, provided the original author(s) and the source are credited.

\section{References}

1. Heikens A, Sumarti S, van Bergen M, Widianarko B, Fokkert L, van Leeuwen K, Seinen W (2005) The impact of the hyperacid Ijen Crater Lake: risks of excess fluoride to human health. Sci Total Environ 346:56-69

2. Gbadebo AM (2012) Groundwater fluoride and dental fluorosis in southwestern Nigeria. Environ Geochem Health 34:597-604

3. Gupta SK, Gambhir S, Mithal A, Das BK (1993) Skeletal scintigraphic findings in endemic skeletal fluorosis. Nucl Med Commun 14:384-390

4. Kierdorf H, Kierdorf U, Richards A, Sedlacek F (2000) Disturbed enamel formation in wild boars (Sus scrofa L.) from fluoride polluted areas in Central Europe. Anat Rec 259:12-24

5. Bernstein DS, Sadowsky N, Hegsted DM, Guri CD, Stare FJ (1966) Prevalence of osteoporosis in high- and low-fluoride areas in North Dakota. JAMA 198:499-504

6. Palmer C, Wolfe SH (2005) Position of the American Dietetic Association: the impact of fluoride on health. J Am Diet Assoc 105:1620-1628

7. Kleerekoper M, Peterson EL, Nelson DA, Phillips E, Schork MA, Tilley BC, Parfitt AM (1991) A randomized trial of sodium fluoride as a treatment for postmenopausal osteoporosis. Osteoporos Int 1: $155-161$

8. Riggs BL, Hodgson SF, O'Fallon WM, Chao EY, Wahner HW, Muhs JM, Cedel SL, Melton LJ 3rd (1990) Effect of fluoride treatment on the fracture rate in postmenopausal women with osteoporosis. N Engl J Med 322:802-809

9. Boivin G, Chavassieux P, Chapuy MC, Baud CA, Meunier PJ (1989) Skeletal fluorosis: histomorphometric analysis of bone changes and bone fluoride content in 29 patients. Bone 10:89-99

10. Fratzl P, Roschger P, Eschberger J, Abendroth B, Klaushofer K (1994) Abnormal bone mineralization after fluoride treatment in osteoporosis: a small-angle x-ray-scattering study. J Bone Miner Res 9:1541-1549

11. Gordon SL, Corbin SB (1992) Summary of workshop on drinking water fluoride influence on hip fracture on bone health. (National Institutes of Health, 10 April, 1991). Osteoporos Int 2:109-117

12. Jacobsen SJ, Goldberg J, Miles TP, Brody JA, Stiers W, Rimm AA (1990) Regional variation in the incidence of hip fracture. US white women aged 65 years and older. JAMA 264:500-502

13. Cooper C, Wickham CA, Barker DJ, Jacobsen SJ (1991) Water fluoridation and hip fracture. JAMA 266:513-514

14. Beltran-Neira RJ, Beltran-Aguilar ED (2004) Taxonomy for competency-based dental curricula. J Dent Educ 68:978-984

15. K Sebelius (2011) Proposed HHS Recommendation for Fluoride Concentration in Drinking Water for Prevention of Dental Caries. In Services HaH (ed.) Federal Register, pp. 2383-2388

16. Busse B, Jobke B, Hahn M, Priemel M, Niecke M, Seitz S, Zustin J, Semler J, Amling M (2010) Effects of strontium ranelate administration on bisphosphonate-altered hydroxyapatite: matrix incorporation of strontium is accompanied by changes in mineralization and microstructure. Acta Biomater 6:4513-4521

17. Milovanovic P, Zimmermann EA, Hahn M, Djonic D, Puschel K, Djuric M, Amling M, Busse B (2013) Osteocytic canalicular networks: morphological implications for altered mechanosensitivity. ACS Nano

18. Roschger P, Plenk H Jr, Klaushofer K, Eschberger J (1995) A new scanning electron microscopy approach to the quantification of bone mineral distribution: backscattered electron image grey-levels correlated to calcium K alpha-line intensities. Scanning Microsc 9:75-86, discussion 86-78

19. Roschger P, Fratzl P, Eschberger J, Klaushofer K (1998) Validation of quantitative backscattered electron imaging for the measurement of mineral density distribution in human bone biopsies. Bone 23:319-326

20. Roschger P, Paschalis EP, Fratzl P, Klaushofer K (2008) Bone mineralization density distribution in health and disease. Bone 42:456-466

21. Seitz S, Koehne T, Ries C, De Novo OA, Barvencik F, Busse B, Eulenburg C, Schinke T, Puschel K, Rueger JM, Amling M, Pogoda P (2013) Impaired bone mineralization accompanied by low vitamin $\mathrm{D}$ and secondary hyperparathyroidism in patients with femoral neck fracture. Osteoporos Int 24:641-649

22. Koehne T, Marshall RP, Jeschke A, Kahl-Nieke B, Schinke T, Amling M (2013) Osteopetrosis, osteopetrorickets and hypophosphatemic rickets differentially affect dentin and enamel mineralization. Bone 53:25-33

23. Beil FT, Oheim R, Barvencik F, Hissnauer TN, Pestka JM, Ignatius A, Rueger JM, Schinke T, Clarke IJ, Amling M, Pogoda P (2012) Low turnover osteoporosis in sheep induced by hypothalamicpituitary disconnection. J Orthop Res 30:1254-1262

24. Mathey J, Horcajada-Molteni MN, Chanteranne B, Picherit C, Puel C, Lebecque P, Cubizoles C, Davicco MJ, Coxam V, Barlet JP (2002) Bone mass in obese diabetic Zucker rats: influence of treadmill running. Calcif Tissue Int 70:305-311

25. Amling M, Priemel M, Holzmann T, Chapin K, Rueger JM, Baron R, Demay MB (1999) Rescue of the skeletal phenotype of vitamin D receptor-ablated mice in the setting of normal mineral ion homeostasis: formal histomorphometric and biomechanical analyses. Endocrinology 140:4982-4987

26. Parfitt AM, Drezner MK, Glorieux FH, Kanis JA, Malluche H, Meunier PJ, Ott SM, Recker RR (1987) Bone histomorphometry: standardization of nomenclature, symbols, and units. Report of the ASBMR Histomorphometry Nomenclature Committee. J Bone Miner Res 2:595-610

27. Regelsberger J, Milovanovic P, Schmidt T, Hahn M, Zimmermann EA, Tsokos M, Zustin J, Ritchie RO, Amling M, Busse B (2012) Changes to the cell, tissue and architecture levels in cranial suture synostosis reveal a problem of timing in bone development. Eur Cell Mater 24:441-458

28. Kubek DJ, Gattone VH 2nd, Allen MR (2010) Methodological assessment of acid-etching for visualizing the osteocyte lacunarcanalicular networks using scanning electron microscopy. Microsc Res Tech 73:182-186

29. Oheim R, Beil FT, Kohne T, Wehner T, Barvencik F, Ignatius A, Amling M, Clarke IJ, Pogoda P (2013) Sheep model for osteoporosis: sustainability and biomechanical relevance of low turnover osteoporosis induced by hypothalamic-pituitary disconnection. J Orthop Res 31:1067-1074

30. Buchancova J, Polacek H, Hudeckova H, Murajda L, Osina O, Valachova J (2008) Skeletal fluorosis from the point of view of an occupational exposure to fluorides in former Czechoslovakia. Interdiscip Toxicol 1:193-197

31. Kierdorf U, Kierdorf H, Sedlacek F, Fejerskov O (1996) Structural changes in fluorosed dental enamel of red deer (Cervus elaphus L.) from a region with severe environmental pollution by fluorides. J Anat 188(Pt 1):183-195 
32. Kakumanu N, Rao SD (2013) Images in clinical medicine. Skeletal fluorosis due to excessive tea drinking. N Engl J Med 368:1140

33. Cao JLJ, Tang LL, Sangbu DZ, Yu S, Zhou S, Yu Y, Qu HY (2005) Dental and early-stage skeletal fluorosis in children induced by fluoride in brick-tea. Fluoride 38:44-47

34. Fejerskov OMF, Baelum V (1990) The nature and mechanisms of dental fluorosis in man. J Dent Res 69:692-700, discussion 721

35. Wang W, Kong L, Zhao H, Dong R, Li J, Jia Z, Ji N, Deng S, Sun Z, Zhou J (2007) Thoracic ossification of ligamentum flavum caused by skeletal fluorosis. Eur Spine J 16:1119-1128

36. Medina-Solis CE, Pontigo-Loyola AP, Maupome G, LamadridFigueroa H, Loyola-Rodriguez JP, Hernandez-Romano J, Villalobos-Rodelo JJ, de Lourdes M-CM (2008) Dental fluorosis prevalence and severity using Dean's index based on six teeth and on 28 teeth. Clin Oral Investig 12:197-202

37. Stevenson CA, Watson AR (1957) Fluoride osteosclerosis. Am J Roentgenol Radium Ther Nucl Med 78:13-18

38. Schlesinger ER, Overton DE, Chase HC, Cantwell KT (1956) Newburgh-Kingston caries-fluorine study. XIII. Pediatric findings after ten years. J Am Dent Assoc 52:296-306

39. Mc CH, Mc CF (1954) Effect of fluoride in drinking water on the osseous development of the hand and wrist in children. Public Health Rep 69:671-683

40. Sowers MR, Wallace RB, Lemke JH (1986) The relationship of bone mass and fracture history to fluoride and calcium intake: a study of three communities. Am J Clin Nutr 44:889-898

41. Kaminsky LS, Mahoney MC, Leach J, Melius J, Miller MJ (1990) Fluoride: benefits and risks of exposure. Crit Rev Oral Biol Med 1: 261-281

42. Schinke T, Schilling AF, Baranowsky A, Seitz S, Marshall RP, Linn T, Blaeker M, Huebner AK, Schulz A, Simon R, Gebauer M, Priemel
M, Kornak U, Perkovic S, Barvencik F, Beil FT, Del Fattore A, Frattini A, Streichert T, Pueschel K, Villa A, Debatin KM, Rueger JM, Teti A, Zustin J, Sauter G, Amling M (2009) Impaired gastric acidification negatively affects calcium homeostasis and bone mass. Nat Med 15:674-681

43. Jacks G, Rajagoplan K, Alveteg T, Jonsson M (1993) Genesis of high-F groundwaters, southern India. Appl Geochem 8: 241-244

44. Zheng BS, Dingam ZH, Huang RG, Zhu JM, Yu XY, Wang AM, Zhou DX, Mao DJ, Su HC (1999) Issues of health and disease relating to coal use in southwestern China. Int J Coal Geol 40:119-132

45. Grynpas MD, Holmyard D, Pritzker KP (1994) Bone mineralization and histomorphometry in biopsies of patients treated with fluoride. Cells Mater 4:287-297

46. Baylink D, Wergedal J (1970) Effects of fluoride on bone formation, mineralization, and resorption in the rat. In: Vischer TL (ed) Fluoride in medicine. Hans Huber Publisher, Bern, pp 37-69

47. Riggs BL (1983) Treatment of osteoporosis with sodium fluoride: an appraisal. In: Peck WA (ed) Bone and mineral research. Elsevier, Amsterdam, pp 366-393

48. Dittmer KE, Thompson KG, Blair HT (2009) Pathology of inherited rickets in Corriedale sheep. J Comp Pathol 141:147-155

49. Oheim R, Amling M, Ignatius A, Pogoda P (2012) Large animal model for osteoporosis in humans: the ewe. Eur Cell Mater 24:372385

50. Priemel M, von Domarus C, Klatte TO, Kessler S, Schlie J, Meier S, Proksch N, Pastor F, Netter C, Streichert T, Puschel K, Amling M (2010) Bone mineralization defects and vitamin D deficiency: histomorphometric analysis of iliac crest bone biopsies and circulating 25-hydroxyvitamin D in 675 patients. J Bone Miner Res 25:305312 